\title{
Prediction of protein complex structure using surface-induced dissociation and cryo-EM
}

Justin T. Seffernick, ${ }^{1}$ Shane M. Canfield, ${ }^{2}$ Sophie R. Harvey, ${ }^{1}$ Vicki H. Wysocki, ${ }^{1}$ and Steffen Lindert ${ }^{1, *}$

${ }^{1}$ Department of Chemistry and Biochemistry, Ohio State University, Columbus, OH, 43210

${ }^{2}$ Department of Chemistry, Kenyon College, Gambier, OH, 43022

* Correspondence to:

Department of Chemistry and Biochemistry, Ohio State University

2114 Newman \& Wolfrom Laboratory, 100 W. $18^{\text {th }}$ Avenue, Columbus, OH 43210

614-292-8284 (office), 614-292-1685 (fax)

lindert.1@,osu.edu

\section{Supplementary Information}

\section{Methods}

\section{Cryo-EM density map simulation}

Low-resolution cryo-EM density maps were simulated from crystal structures using BCL::density::FromPDB ${ }^{1}$ at a resolution of $14 \AA$ and a voxel size of $1.4 \AA$. In order to mimic the inherent error present in experimental density maps and thus simulate more realistic maps, Gaussian noise was included in these simulations..$^{2-4}$ Noise was randomly added until the crosscorrelation coefficient between the noise-containing and noise-free maps was $\sim 0.8$ as has been previously demonstrated to be realistic. ${ }^{1}$ Density maps were simulated for all complexes and were used for scoring.

\section{Input structures for docking}

First, we built homology models (HM) of monomers when possible. Accurate tertiary structures (RMSD $<2 \AA$ ) were built for the monomers of $1 \mathrm{GNH}, 1 \mathrm{GZX}$ (both chain A and B), 1SAC, 1SWB, and 8TIM with multi-template homology modeling using RosettaCM. All Rosetta calculations were performed using the talaris2014 scoring function. The templates used for HM were 4PBO (34\% identity, 56\% similarity), $3 \mathrm{GO} 6(31 \%, 55 \%)$, and $3 \mathrm{HX} 6(26 \%, 45 \%)$ for $1 \mathrm{GNH}$; 2LHB $(32 \%, 50 \%)$, 2NRL $(32 \%, 44 \%)$, and $1 \mathrm{MYT}(27 \%, 41 \%)$ for $1 \mathrm{GZX}$ chain A; $1 \mathrm{MYT}(33 \%$, 46\%), 3QMA (33\%, 45\%), and 1LHS (31\%, 48\%) for $1 \mathrm{GZX}$ chain B; 4PBO $(39 \%, 54 \%)$, 4PBP $(39 \%, 54 \%)$, and 4QVS $(22 \%, 38 \%)$ for $1 \mathrm{SAC}$; 3RE6 $(96 \%, 97 \%), 3 \mathrm{RDM}(95 \%, 96 \%)$, and $4 \mathrm{JNJ}$ $(59 \%, 63 \%)$ for $1 \mathrm{SWB}$; and 2WSR $(51 \%, 64 \%)$, 1TRI $(50 \%, 64 \%)$, and 2 VEK $(48 \%, 61 \%)$ for $8 \mathrm{TIM}$. For each monomer, 10,000 structures were built using RosettaCM, with each template weighted equally, and the predicted monomers used for docking were the lowest scoring models. Next, we used unbound input structures, crystal structures of a monomer or subcomplex separate from the full complex, for two proteins for which the structures were available: hemoglobin (heterodimer, 2HBC) and streptavidin (monomer, 5N8T). Finally, we perturbed the bound structures for all benchmark complexes prior to input in docking using Rosetta relax, normal mode analysis, and backrub.

\section{Analysis metrics}

The RMSD (root-mean-square deviation) to native was calculated for both types of docking to evaluate the results. All atom, aligned RMSD with refinement was calculated using PyMol. ${ }^{5} \mathrm{RMSD}_{100}$, a normalized RMSD value relating the RMSD to that of a 100 residue protein, 
was also calculated. ${ }^{6} \mathrm{CAPRI}$ protein-protein docking model quality metrics $\left(\mathrm{F}_{\text {nat }}, \mathrm{F}_{\text {non-nat, }}, \mathrm{iRMSD}\right.$, LRMSD, and DockQ score) were calculated using DockQ. ${ }^{7}$ Frequency of native contacts $\left(\mathrm{F}_{\text {nat }}\right)$ was defined as the ratio of contacts across the interface compared to those in the native, with residue-residue contacts defined if any heavy atoms were within $5 \AA$. Frequency of non-native contacts ( $\left.F_{\text {non-nat }}\right)$ was defined as the ratio of contacts (defined similarly) in the model not observed in the native structure. Interface RMSD (iRMSD) was defined as heavy atom RMSD of interface residues (residues with any heavy atom within $10 \AA$ of the adjacent chain). Ligand RMSD (LRMSD) was defined as backbone RMSD of the smaller chain when larger chains were aligned. Finally, DockQ score is a metric, ranging between 0 and 1 , used to quantify model quality in a similar fashion to CAPRI. Models were classified as incorrect if the DockQ score was less than 0.23 , acceptable if the DockQ score was less than 0.49, but greater than 0.23, medium if the DockQ score was less than 0.80, but greater than 0.49 , and high if the DockQ score was greater than 0.80 . CAPRI model quality was also calculated as described previously. ${ }^{8}$ For symmetric docking, the DockQ scores (and other metrics calculated using DockQ) were calculated only on the interfaces built and scored with SID. If multiple SID-scored interfaces were built in a single symmetric dock, the results were averaged. Finally, $\mathrm{P}_{\text {near }},{ }^{9}$ quantifying the goodness of funneling of the score vs. RMSD distribution was calculated (after normalization for fair comparison between different weights) using the following parameters: lambda of 4.0 and $\mathrm{k}_{\mathrm{B}} \mathrm{T}$ of 0.005 . 
Table S1: List of interfaces built for the ensemble docking. Chains are provided in the format $\mathrm{X}_{-} \mathrm{Y}$, where $\mathrm{X}$ is the chain ID of the stationary chain and $\mathrm{Y}$ is the chain ID for the mobile chain. $\mathrm{X}_{-} \mathrm{Y}$ also corresponds to the input into docking via the partners flag. For complex type, whole complexes are shown, but portions built during each docking are shown in color. Types of input structures are also shown: $\mathrm{HM}=$ homology model, unbound $=$ crystal structure of the monomer/subcomplex separately, bound + perturbed $=$ monomers $/$ subcomplexes obtained from the full complex crystal structure.

\begin{tabular}{|c|c|c|c|c|}
\hline PDB & Input type & Chains & Complex type & Structure \\
\hline $1 \mathrm{GNH}$ & $\mathrm{HM}$ & A_B & & \\
\hline 1GZX & HM & A_B & & \\
\hline $1 \mathrm{SAC}$ & HM & A_B & & \\
\hline 1SWB & HM & A_B & & \\
\hline 8TIM & HM & A_B & & \\
\hline $2 \mathrm{HBC}(1 \mathrm{GZX})$ & Unbound & AB_CD & & \\
\hline 5N8T (1SWB) & Unbound & A_B & & \\
\hline 1FGB & Bound+perturbed & A_B & & \\
\hline $1 \mathrm{GNH}$ & Bound +perturbed & A_B & & \\
\hline $1 \mathrm{GZX}$ & Bound+perturbed & A_B & & a) \\
\hline & & & & \\
\hline
\end{tabular}




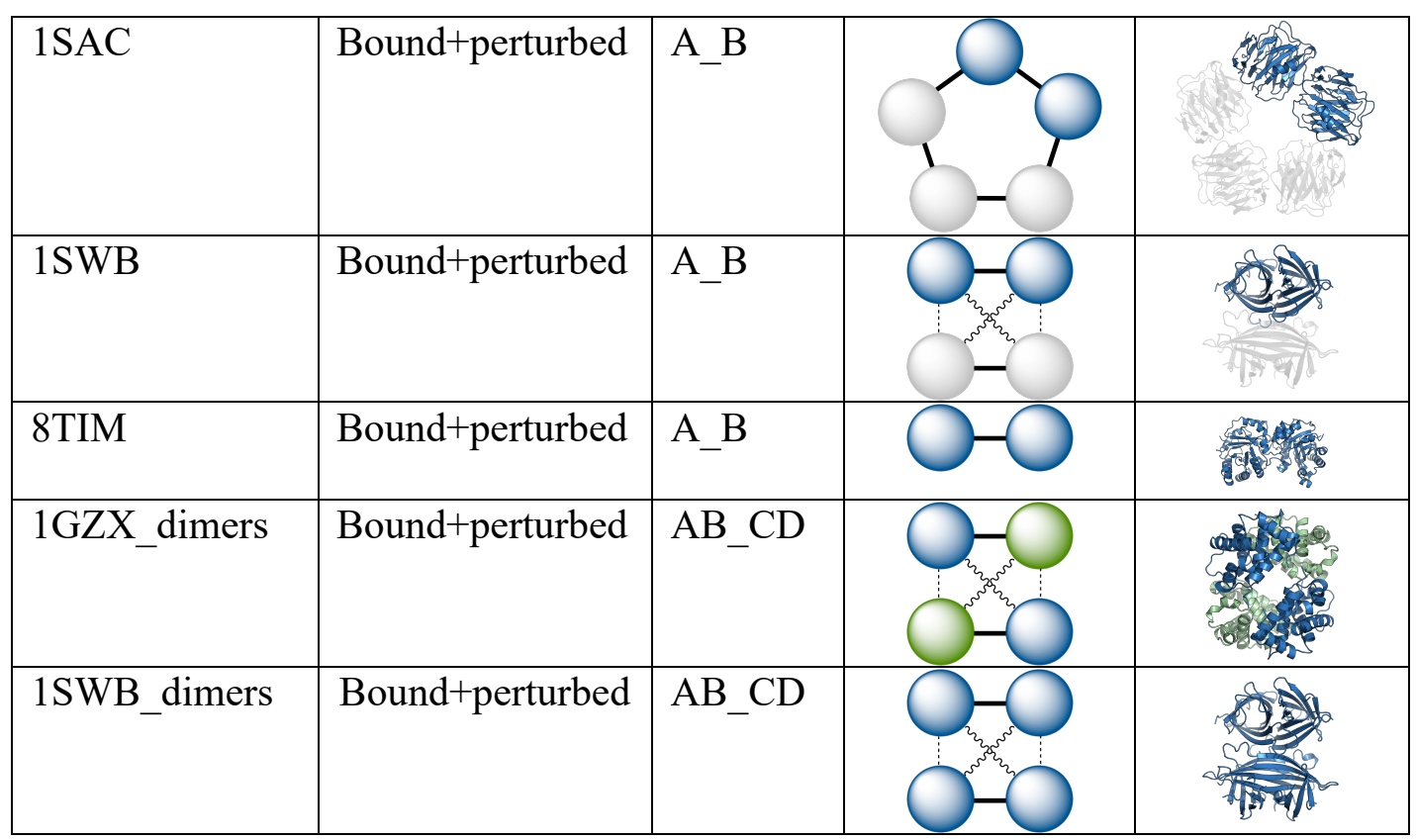


Table S2: List of symmetries for the symmetric docking. De novo symmetry was used for all complexes with the exception of streptavidin (1SWB and 5N8T), for which noncrystallographic point symmetry was used. Input structures for complexes indicated with an asterisk were first created using ensemble docking. For symmetric docking, full complexes were built in all cases. Types of input structures are also shown: HM = homology model, unbound = crystal structure of the monomer/subcomplex separately, bound + perturbed $=$ monomers/subcomplexes obtained from the full complex crystal structure.

\begin{tabular}{|c|c|c|c|c|}
\hline PDB & Input type & Symmetry & Complex type & Structure \\
\hline $1 \mathrm{GNH}$ & $\mathrm{HM}$ & $\mathrm{C} 5$ & & \\
\hline 1GZX (AB)* & HM & $\mathrm{C} 2$ & & \\
\hline $1 \mathrm{SAC}$ & HM & $\mathrm{C} 5$ & & \\
\hline $1 \mathrm{SWB}$ & HM & D2 (NCS) & & \\
\hline $8 \mathrm{TIM}$ & HM & $\mathrm{C} 2$ & & \\
\hline $2 \mathrm{HBC}(\mathrm{AB}, 1 \mathrm{GZX})$ & Unbound & $\mathrm{C} 2$ & & \\
\hline 5N8T (1SWB) & Unbound & D2 (NCS) & & \\
\hline 1FGB & Bound+perturbed & $\mathrm{C} 5$ & & \\
\hline $1 \mathrm{GNH}$ & Bound+perturbed & $\mathrm{C} 5$ & & \\
\hline 1GZX (AB)* & Bound + perturbed & $\mathrm{C} 2$ & & \\
\hline
\end{tabular}




\begin{tabular}{|l|l|l|l|l|}
\hline 1SAC & Bound+perturbed & C5 & & \\
\hline $1 \mathrm{SWB}$ & Bound+perturbed & $\mathrm{D} 2$ (NCS) & & \\
\hline 1GTIM & Bound+perturbed & $\mathrm{C} 2$ & & \\
\hline 1SWB_dimers & Bound+perturbed & $\mathrm{C} 2$ & & \\
& & & $\mathrm{C} 2$ & \\
\hline
\end{tabular}

Table S3: Relative weights used for scoring with the combined scores (Isc+cryo-EM and Isc+cryo-EM+SID) after normalization of each individual term for each protein. Note: crystal refers to both unbound and perturbed+bound crystal structures.

\begin{tabular}{|l|c|c|c|c|}
\hline \multicolumn{1}{|c|}{ Term } & Ensemble HM & Ensemble crystal & Symmetric HM & Symmetric crystal \\
\hline Isc & 1 & 5 & 1 & 1 \\
\hline Cryo-EM & 28 & 7 & 16 & 16 \\
\hline SID & 5 & 1 & 35 & 30 \\
\hline
\end{tabular}


Table S4: Averages of metrics used to quantify docking results for the predicted structures using Isc, Isc+cryo-EM, and combined Isc+cryo-EM+SID. Average quality (A: acceptable, M: medium) was determined based on average DockQ score. Metrics generally improved when all experimental data were included (left to right).

\begin{tabular}{|l|c|c|c|c|c|c|}
\hline & \multicolumn{3}{|c|}{ Ensemble docking } & \multicolumn{3}{c|}{ Symmetric docking } \\
\hline Metric & Isc & $\begin{array}{c}\text { Isc } \\
\text { cryo-EM }\end{array}$ & $\begin{array}{c}\text { Isc } \\
+ \text { cryo-EM } \\
+ \text { SID }\end{array}$ & Isc & $\begin{array}{c}\text { Isc } \\
+ \text { cryo-EM }\end{array}$ & $\begin{array}{c}\text { Isc } \\
+ \text { cryo-EM } \\
+ \text { SID }\end{array}$ \\
\hline RMSD $(\AA)$ & 7.35 & 3.61 & 1.92 & 6.59 & 2.13 & 1.71 \\
\hline RMSD $_{100}(\AA)$ & 4.41 & 2.23 & 1.15 & 12.56 & 4.33 & 3.38 \\
\hline $\mathrm{F}_{\text {nat }}$ & 0.27 & 0.44 & 0.54 & 0.23 & 0.49 & 0.46 \\
\hline $\mathrm{F}_{\text {non-nat }}$ & 0.66 & 0.43 & 0.30 & 0.71 & 0.38 & 0.36 \\
\hline iRMSD $(\AA)$ & 8.82 & 4.35 & 2.16 & 10.90 & 3.09 & 2.94 \\
\hline LRMSD $(\AA)$ & 23.90 & 12.66 & 4.74 & 26.78 & 8.52 & 6.89 \\
\hline DockQ score & 0.30 & 0.48 & 0.57 & 0.26 & 0.54 & 0.52 \\
\hline Quality & $\mathrm{A}$ & $\mathrm{A}$ & $\mathrm{M}$ & $\mathrm{A}$ & $\mathrm{M}$ & $\mathrm{M}$ \\
\hline
\end{tabular}

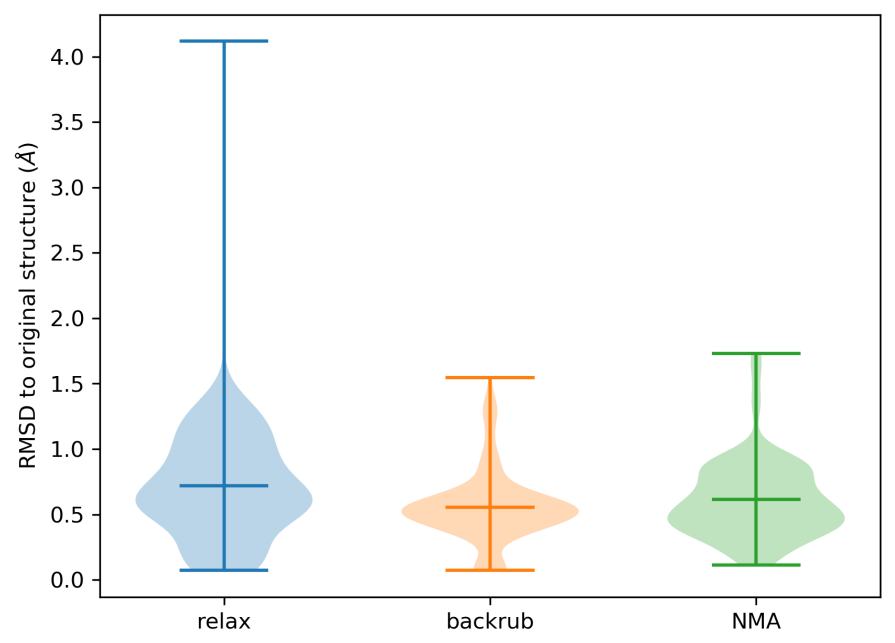

Figure S1: Frequency of RMSD to the input monomers for the backbone sampling methods of Rosetta relax, normal mode analysis, and backrub. 

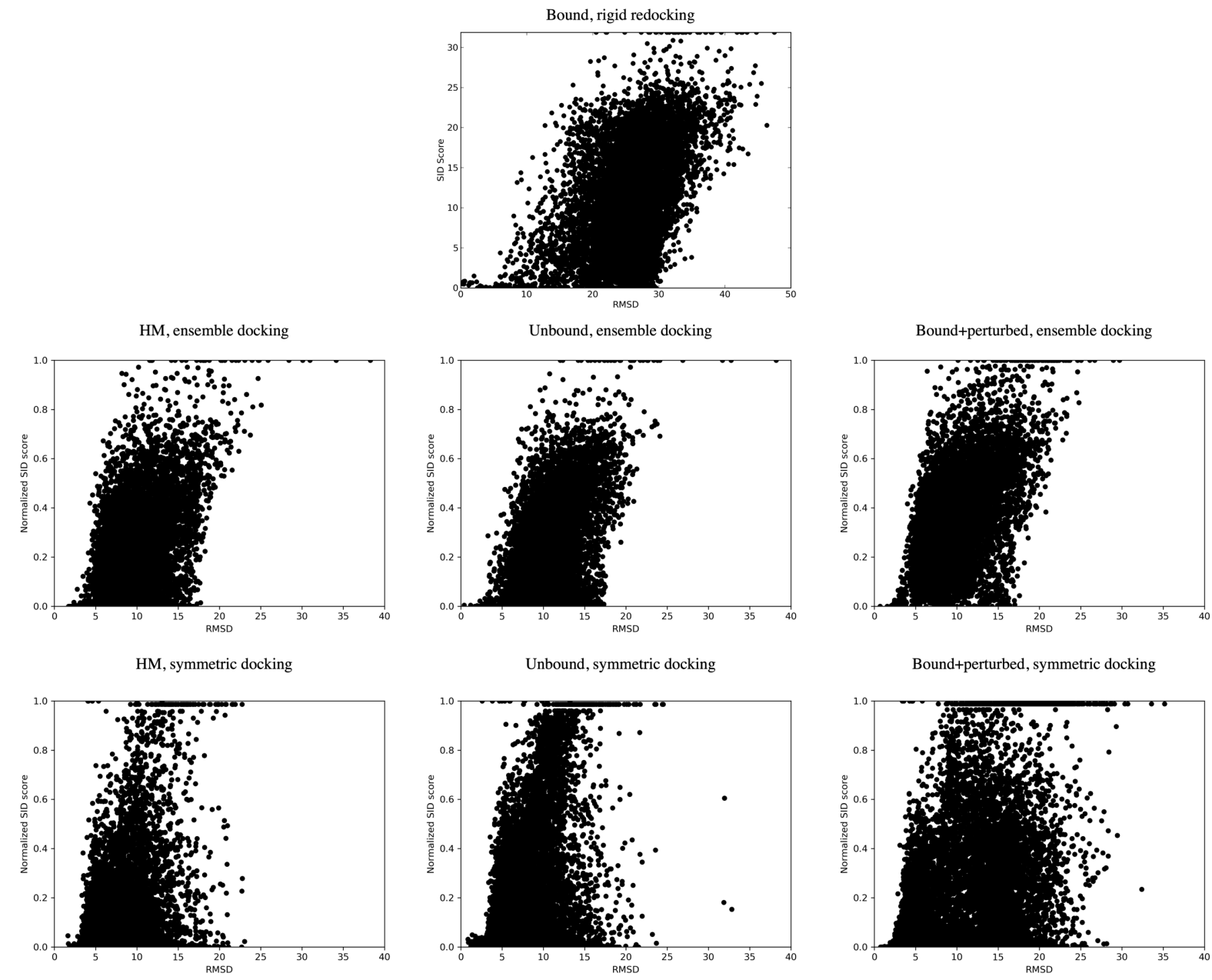

Figure S2: SID score vs. RMSD plots for 1SWB: bound rigid redocking (previous work. Note: SID scores were un-normalized and RMSDs were LRMSD rather than aligned) ${ }^{10} \mathrm{HM}$ ensemble docking, unbound (5N8T) ensemble docking, perturbed+bound ensemble docking, HM symmetric docking, unbound (5N8T) symmetric docking, and perturbed+bound symmetric docking.
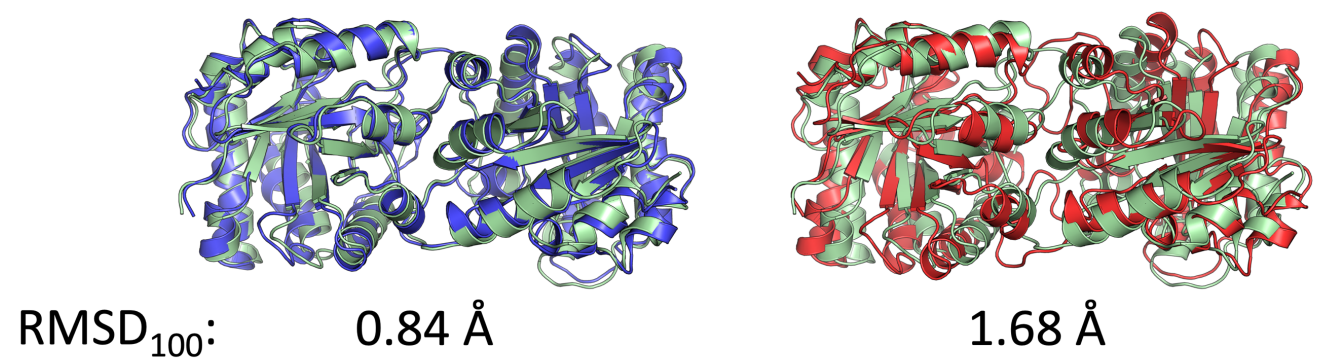

Figure S3: Symmetric docked structures for 8TIM. The structure predicted from Isc + cryo-EM is shown in blue, Isc+cryo-EM+SID shown in red, and native shown in green. Although CASP metrics appear slightly worse for 8TIM, the structures are very similar. 


\section{Tutorial:}

This tutorial contains example Rosetta (and BCL) commands to run the calculations and scoring described in this manuscript.

\section{Simulating density maps (using BCL):}

/bcl-3.6.1-Source/build/linux64_release/bin/bcl-apps-static.exe density:FromPDB complex_pdb_file.pdb -resolution 14.0 -voxel_size 1.4 kernel GaussianSphere -noise 0.8 -random_seed

Note: complex_pdb_file.pdb is the pdb file of the complex that you will generate the density map for. The density map will be used for scoring in this tutorial.

Also note: resolution, voxel size (set to $1 / 10$ of resolution), and noise level can be changed.

\section{Backbone sampling:}

1. Relax

/Rosetta/main/source/bin/relax.default. $\langle o s\rangle\langle$ compiler $\rangle$ release @flags_relax_mon

In the file flags_relax_mon:

-database $\sim /$ Rosetta/main/database/

-in:file:s subcomplex_pdb_file.pdb

-nstruct 10

-ex 1

-ex2aro

-out:prefix relax

-restore_talaris_behavior

Note: subcomplex_pdb_file.pdb is the input PDB structure to be used for docking. It can be a monomer or a subcomplex.

2. Backrub

/Rosetta/main/source/bin/backrub. default. $\langle$ os $\rangle\langle$ compiler $\rangle$ release @flags_backrub_mon

In the file flags_backrub_mon:

-database /Rosetta/main/database/

-in:file:s subcomplex_pdb_file.pdb

-nstruct 10

-ex 1

-ex2aro

-backrub:mc_kt 0.6

-backrub:ntrials 20000

-out:prefix backrub

-restore_talaris_behavior

3. Normal mode analysis

/Rosetta/main/source/bin/rosetta_scripts.default. $\langle o s\rangle\langle$ compiler $\rangle$ r

elease@flags_nma_mon

In the file flags_nma_mon:

-database $\sim /$ Rosetta/main/database/

-in:file:s subcomplex_pdb_file.pdb

-nstruct 10 


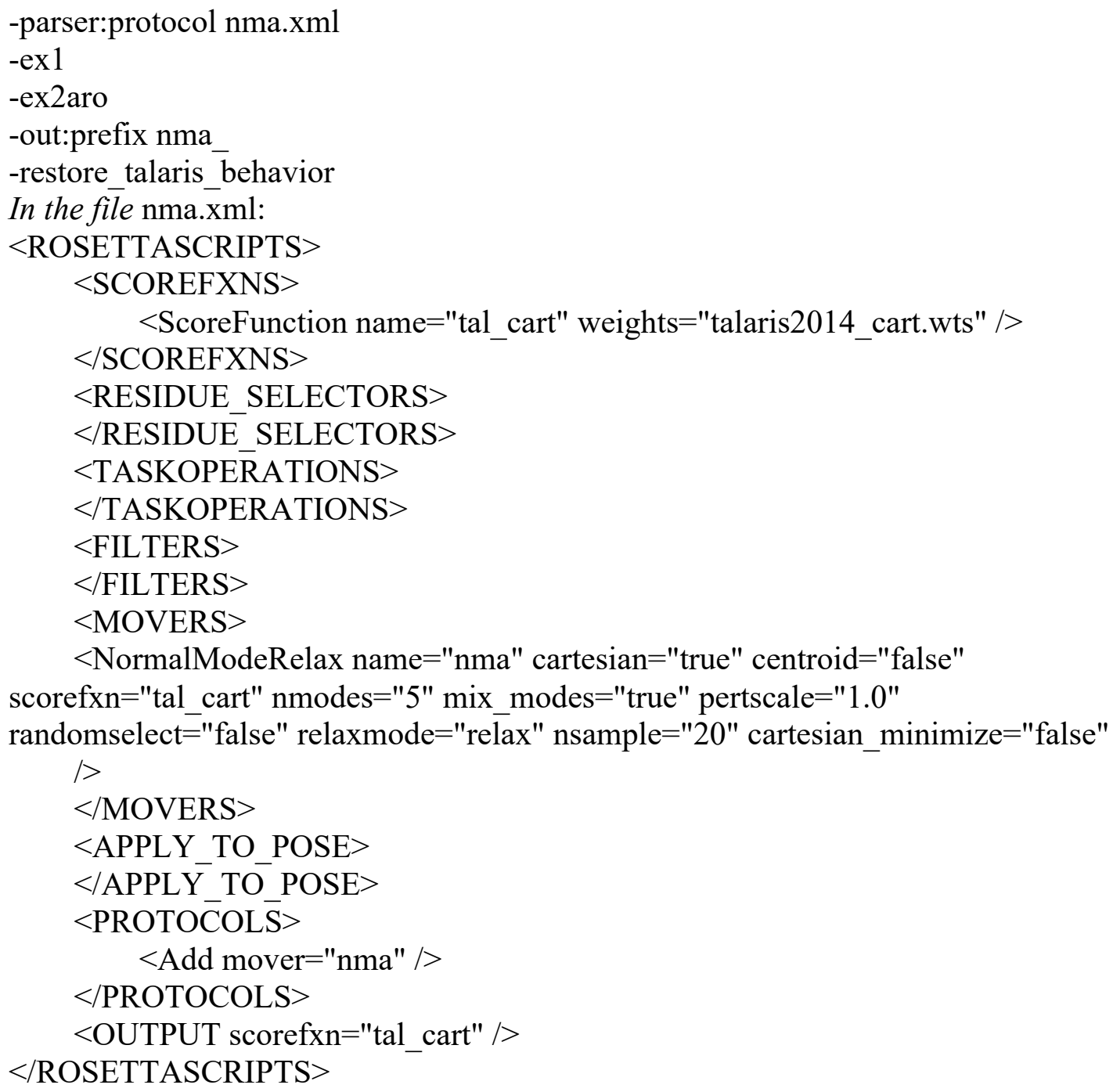

\section{Docking:}

Detailed tutorials for both ensemble and symmetric docking can be found at the following addresses, respectively.

https://www.rosettacommons.org/demos/latest/tutorials/Protein-Protein-Docking/Protein-

Protein-Docking (see Docking Flexible Proteins section)

https://new.rosettacommons.org/docs/latest/application_documentation/docking/sym-dock

Example commands for docking are also provided below.

1. Ensemble docking

Prepack:

/Rosetta/main/source/bin/docking_prepack_protocol.default. $\langle$ os $\rangle\langle c$ ompiler>release@flags_ensemble_prepack

In the file flags_ensemble_prepack:

-database $\sim /$ Rosetta/main/database/

-in:file:s complex_pdb_file.pdb

-nstruct 1

-partners X_Y

-ensemble1 ensemble_1_list 
-ensemble2 ensemble_2_list

$-\mathrm{ex} 1$

-ex2aro

-out:prefix ppk_ensemble

-restore_talaris_behavior

Note: the complex_pdb_file.pdb is the starting structure you wish to dock. You can choose where you want to start the chains. In this tutorial, the randomize2 flag will be used to alter the position of chain 2 prior to docking, thus sampling conformational space.

Also note: $\mathrm{X}_{-} \mathrm{Y}$ represents the partners you wish to dock. $\mathrm{X}$ or $\mathrm{Y}$ can be multiple chains if you wish to dock a subcomplex. For example, docking a dimer to a dimer may be AB_CD. Also note: In files ensemble_1_list and ensemble_2_list, provide the filenames of the backbones sampled for both partners. The ensemble list files are updated after this calculation automatically to reflect the prepacking.

\section{Dock:}

$\sim /$ Rosetta/main/source/bin/docking_protocol.default.〈os $\rangle\langle$ compiler $\rangle$ release flags_ensemble_docking

In the file flags ensemble docking:

-database $\sim /$ Rosetta/main/database/

-in:file:s complex_pdb_file.pdb

-nstruct Z

-partners X_Y

-randomize 2

-ensemble1 ensemble_1_list

-ensemble2 ensemble_2_list

$-\mathrm{ex} 1$

-ex2aro

-out:prefix dock_ensemble

-restore_talaris_behavior

Note: $\mathrm{Z}$ is the number of structures you wish to generate.

2. Symmetric docking

Generate symdef file:

Instructions for generating symmetry definition can be found in the following links:

https://new.rosettacommons.org/docs/latest/application_documentation/utilities/makesymmdef-file

https://www.rosettacommons.org/demos/latest/tutorials/Symmetry/Symmetry

Dock:

/ Rosetta/main/source/bin/SymDock.default. $\langle$ os $\rangle\langle$ compiler $\rangle$ release @flags_sym_dock

In the file flags_sym_dock:

-database $\sim$ Rosetta/main/database/

-in:file:s subcomplex_pdb_file.pdb

-symmetry:symmetry_definition symdef_file.sym

-nstruct $Z$

-symmetry:initialize_rigid_body_dofs

-symmetry:symmetric_rmsd

$-\mathrm{ex} 1$ 
-ex2aro

-out:prefix dock_symmetric_

-out:file:fullatom

-restore_talaris_behavior

Note: run this command for each sampled backbone structure (500 was done for all 30 here). To do this, simply replace subcomplex_pdb_file.pdb with each of the 30 inputs and run separately.

Also note: the syndef_file.sym is the name of the symdef file generated in the previous step.

\section{Scoring and postprocessing:}

1. Interface scores

To obtain interface scores (Isc), simply extract the values from score files.

2. Relax

Prior to calculating cryo-EM scores, SID scores, and RMSD (steps 3-5 below), relax the docked structures.

/Rosetta/main/source/bin/relax.default. $\langle o s\rangle\langle$ compiler $\rangle$ release @flags_relax

In the file flags_relax:

-database $\sim /$ Rosetta/main/database/

-in:file:s docked_pdb_file.pdb

-nstruct 1

-ex 1

-ex2aro

-out:prefix relax

-restore talaris behavior

3. Cryo-EM scores

/Rosetta/main/source/bin/score_jd2.default.linuxgccrelease @flags_cryo-EM_score

In the file flags_cryo-EM_score:

-database $\sim /$ Rosetta/main/database/

-in:file:s relaxed pdb_file.pdb

-ex 1

-ex2aro

-edensity::mapfile density_map_name.mrc

-edensity::mapreso 14.0

-edensity::cryoem_scatterers

-out:prefix cryo-EM_score

-crystal_refine

-restore_talaris_behavior

To obtain the cryo-EM scores, extract the values (elec_dens_fast) from the score files.

Note: the density_map_name.mrc file is the density map generated at the beginning of this tutorial. Alternatively, use a density map obtained from cryo-EM experiments.

4. SID scores

/Rosetta/main/source/bin/SID_rescore.default. $\langle 0 s\rangle\langle$ compiler $\rangle$ relea se @flags_SID_score

In the file flags SID score:

-in:file:1 list_of_relaxed_docked_pdbs 
-AE appearance_energy_value

-interface X_Y

-n_ints number_of_interfaces

-out:file:o SID_score_output_file_name

-skip_relax

To obtain SID scores, extract the values (SID_score) from the output file.

Note: a more in-depth tutorial for SID scoring was provided previously. ${ }^{10}$ In short: list_of_relaxed_docked_pdbs is a file containing the names of the relaxed, docked pdb files (alternatively, run separately using -in:file:s for each pdb), appearance_energy_value is the AE obtained from SID experiments, number_of_interfaces is the number of protein-protein interfaces corresponding to the AE, and SID_score_output_file_name is the name of the output file for the scores.

5. Calculate combined scores

To calculate the combined scores (Isc+cryo-EM and Isc+cryo-EM+SID), first normalize each individual score for each protein. To do this, obtain the most extreme value for each protein (most negative for Isc and cryo-EM, most positive for SID). Then, divide each score by that value for each protein. Finally, calculate the combined scores as a linear combination of the terms using the provided weights. 


\section{References}

1. Woetzel, N.; Lindert, S.; Stewart, P. L.; Meiler, J., BCL::EM-Fit: rigid body fitting of atomic structures into density maps using geometric hashing and real space refinement. $J$ Struct Biol 2011, 175 (3), 264-76.

2. $\quad$ Lindert, S.; Alexander, N.; Wötzel, N.; Karakaş, M.; Stewart, P. L.; Meiler, J., EM-fold: de novo atomic-detail protein structure determination from medium-resolution density maps. Structure 2012, 20 (3), 464-78.

3. Leelananda, S. P.; Lindert, S., Iterative Molecular Dynamics-Rosetta Membrane Protein Structure Refinement Guided by Cryo-EM Densities. J Chem Theory Comput 2017, 13 (10), 51315145.

4. Leelananda, S. P.; Lindert, S., Using NMR Chemical Shifts and Cryo-EM Density Restraints in Iterative Rosetta-MD Protein Structure Refinement. J Chem Inf Model 2020, 60 (5), 2522-2532.

5. The PyMOL Molecular Graphics System, Version 2.0 Schrödinger, LLC.

6. Carugo, O.; Pongor, S., A normalized root-mean-square distance for comparing protein three-dimensional structures. Protein science : a publication of the Protein Society 2001, 10 (7), 1470-1473.

7. Basu, S.; Wallner, B., DockQ: A Quality Measure for Protein-Protein Docking Models. PLoS One 2016, 11 (8), e 0161879.

8. Lensink, M. F.; Méndez, R.; Wodak, S. J., Docking and scoring protein complexes: CAPRI 3rd Edition. Proteins 2007, 69 (4), 704-18.

9. $\quad$ Bhardwaj, G.; Mulligan, V. K.; Bahl, C. D.; Gilmore, J. M.; Harvey, P. J.; Cheneval, O.; Buchko, G. W.; Pulavarti, S. V.; Kaas, Q.; Eletsky, A.; Huang, P. S.; Johnsen, W. A.; Greisen, P. J.; Rocklin, G. J.; Song, Y.; Linsky, T. W.; Watkins, A.; Rettie, S. A.; Xu, X.; Carter, L. P.; Bonneau, R.; Olson, J. M.; Coutsias, E.; Correnti, C. E.; Szyperski, T.; Craik, D. J.; Baker, D., Accurate de novo design of hyperstable constrained peptides. Nature 2016, 538 (7625), 329-335.

10. Seffernick, J. T.; Harvey, S. R.; Wysocki, V. H.; Lindert, S., Predicting Protein Complex Structure from Surface-Induced Dissociation Mass Spectrometry Data. ACS Cent Sci 2019, 5 (8), 1330-1341. 